\title{
Living-donor liver transplantation for Abernethy malformation - case report and review of literature
}

\author{
Hoang Duc Nam
}

\author{
HBP-Liver Transplant Center, Vinmec Times City International Hospital, Hanoi, Vietnam
}

\begin{abstract}
Abernethy malformation was named for the rare congenital absence of the portal vein (CAPV), also known as congenital extrahepatic portal-systemic shunts (CEPS). This could be classified as complete (type 1) or incomplete shunt (type 2) according to Morgan-Superina classification. Its presentation may show under variable signs and symptoms such as hepatopulmonary syndrome, hyper-ammonia, hepatic masses and liver failure $\cdots$. This usually combined with other congenital anomalies (cardiac anomaly, trisomy $\cdots$ ). This report presented a 10 year-old boy with growth retardation and mild mental recognition, intermittent hyperammonia, elevated liver enzymes, huge inoperable mass in the right liver. MS CT and MRI findings: hyperplasia of liver parenchyma with superior mesenteric vein confluenced with splenic vein before draining directly into the inferior vena cava (Abernethy anomaly type 1b). Living donor liver transplantation underwent using right lobe from his mother. Anatomopathology findings of the native liver showed chronic hepatitis with cirrhosis $4 / 6$ Knodel-Ishak. Postoperatively, the patient still attained optimal liver function and has returned to normal life at 12-month follow-up. Liver transplantation was a reasonable indication for CAPV type 1. Living donor liver transplantation was effective and practical in the scarcity of donor organ. (Ann Hepatobiliary Pancreat Surg 2020;24:203-208)
\end{abstract}

Key Words: Abernethy malformation; Congenital absence of portal vein; Congenital extrahepatic portosystemic shunt; Living donor liver transplantation

\section{INTRODUCTION}

Congenital absence of portal vein (CAPV), also known as congenital extrahepatic portosystemic shunt (CEPS) was first described by John Abernethy in 1793 in London, England. It was a 10-month old girl who died of unknown reason. On postmortem examination, many congenital anomalies were found with dextrocardia, great vessel transpositions, polysplenia and portal vein bypass the liver and terminated into the inferior vena cava (IVC) at the renal vein level. ${ }^{1}$ Howard and Davenport ${ }^{2}$ referred the term "Abernethy malformation" for any extrahepatic congenital porto-caval shunt, this diversed visceral blood directly to IVC without passing the liver.

The classification of extrahepatic portal-caval shunts by Morgan-Superina in Table $1^{3}$ was widely accepted.

CAPV once considered very rare congenital anomaly.
Until 1997, Howard and Davenport ${ }^{2}$ reported 5 cases of congenital porto-caval shunt, including one end-to-side shunt which were the 13th of such cases in worldwide literature. Sokollik et al. ${ }^{4}$ (2013) resumed Pubmed reports and found 185 extrahepatic porto-caval shunt, with 103 (56\%) type 1 and $82(44 \%)$ type 2 . Intrahepatic shunt were encountered in 131 patients.

Today, with advanced imaging techniques, the reported number of CAPV has been increasing, though most of these were case or serial report. Futhermore, mass screening for hypergalactosemia in neonates in Japan in the last decades accounted for the early and highest prevalence of CAPV in this country. ${ }^{5-8}$

The diagnosis is often incidental and made primarily by Doppler ultrasonography (US), but computed tomography (CT) or magnetic resonance (MR) are required to classify the shunt and to assess associate anomalies.

Received: August 13, 2019; Revised: January 23, 2020; Accepted: January 29, 2020

Corresponding author: Hoang Duc Nam

HBP-Liver Transplant Center, Vinmec Times City International Hospital, 458 Minh Khai Str., Vinh Tuy, Hai Ba Trung, Hanoi 10000, Vietnam Tel: +84-914-490-441, Fax: +84-24-3974-3557, E-mail: hoangducnamscorp@gmail.com

Copyright (C) 2020 by The Korean Association of Hepato-Biliary-Pancreatic Surgery

This is an Open Access article distributed under the terms of the Creative Commons Attribution Non-Commercial License (http://creativecommons.org/ licenses/by-nc/4.0) which permits unrestricted non-commercial use, distribution, and reproduction in any medium, provided the original work is properly cited. Annals of Hepato-Biliary-Pancreatic Surgery • pISSN: 2508-5778 • elSSN: 2508-5859 
Table 1. Classification of extrahepatic portal-caval shunts after Morgan-Superina ${ }^{3}$

Type 1: end-to-side porto-caval shunt
(complete absence of intrahepatic portal branches).

Type 2: Side-to-side porto-caval shunt.

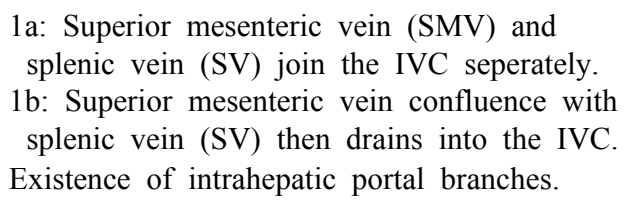

Table 2. Serum biochemical panel follow-up before admission at Vinmec Times City Hospital

\begin{tabular}{llcccc}
\hline Date & NH3 & Bilirubin total/direct & AST/ALT & ALP & GGT \\
\hline 29 Jun 2017 & 73.65 & $29.34 / 14.16$ & $138.51 / 145.31$ & 544.12 & 553.37 \\
13 Jul 2017 & 98.71 & $43.17 / 22.90$ & $87.54 / 77.72$ & - & - \\
24 Aug 2017 & 94.79 & $33.57 / 17.69$ & $154.66 / 155.19$ & - & - \\
21 Sep 2017 & 72.78 & - & $182.82 / 184.64$ & - & - \\
23 Jan 2018 & 69 & $50.2 / 35.5$ & $181 / 207$ & 715 & 435 \\
\hline
\end{tabular}

Reference value according to this referral hospital: NH3 (10-47 mmol/L)

Bilirubin Total (5.13-20.52) and Direct $<3.42 \mathrm{mmol} / \mathrm{L}$

AST 15-60 U/L, ALT 13-45 U/L, ALP 69-362 U/L, GGT $<22 \mathrm{U} / \mathrm{L}$

\section{CASE}

A 10-year old boy transferred to Vinmec Hospital (Hanoi, Vietnam) due to a huge mass on the right liver. His height of $137 \mathrm{~cm}$, weight $24.6 \mathrm{~kg}$, BMI $13.11 \mathrm{~kg} / \mathrm{m}^{2}$. Medical history of congenital cardiac anomaly, diagnosed at 20 days after birth (atrial and ventricular septal defects; pulmonary hypertension up to $50 \mathrm{mmHg}$ ). During childhood he had to admit to hospital frequently (5-6 episodes per year) due to severe pneumonia. Under medical treatment and follow-up, all cardiac septal defects recovered spontaneously and pulmonary hypertension resolved. Since 4 years old, severe pneumonia became less frequent with 1-2 episodes per year. Skeletal anomaly particular noted with agenesis bilateral 5 th toes.

On referral, his serum biochemical panel from previous hospital show intermittent disorders of liver function as in Table 2.

On admission at our hospital, he presented growth retardation and mild mental impairement, transient postprandial drowsiness, and loss of concentration. His height was in standard range, but his weight was $6.1 \mathrm{~kg}$ less than standard reference (comparable with 8 year-old boy) and was evaluated as Tanner stage 1. For mental problem, he had trouble to cope with his friends and stayed at class level 1 elementary school while his friends went to the 4th class. He can still remember simple things like parents name, his home address and so on; but got a long and hard for simple summation (within 10), can only spell but didn't read well. On psychology examination, he was evaluated with Raven Colour Test: IQ 80 (ICD-10-CM: F70 Mild intellectual disabilities) and Vineland Test 90 points.

Clinically, there were abdomen discomfort due to mild hepato-splenomegaly.

His total and direct bilirubin was 49.8 and $24.8 \mu \mathrm{mol} / \mathrm{L}$ (our hospital normal range was $<21$ and 5.1, respectively). AST 133.5 U/L and ALT 135.5 U/L ( $<35$ and 35). GGT: 526 U/L (<55); ALP 676 U/L (30-120). NH3 90.6 $\mu \mathrm{g} / \mathrm{dl}$ (18.7-86.9). AFP $2.29(<9 \mathrm{ng} / \mathrm{ml})$; PIVKA II 81.8 (0-40 $\mathrm{mAU} / \mathrm{ml})$. Liver virus panel and HIV negative. Platelet $326 \mathrm{G} / \mathrm{L}$. INR (International normalized ratio) 1.04. PELD score: 2.8; Child Pugh Score 7 (Grade B). Cardiosonography found no more congenital defects, with EF $68 \%$, without pulmonary hypertension.

Living donor liver transplantation underwent using right lobe from his mother on 22 August 2018. Operation time was 430 minutes, anhepatic phase: 73 minutes. Warm ischemic time (graft out from cold preserve solution for RHV and portal vein reconstruction): 28 minutes; Cold ischemic time: 82 minutes. Portal vein clamping time: 16 minutes. Blood loss $550 \mathrm{ml}$; Red blood cell transfused: $350 \mathrm{ml}$. Graft weight: 549 gr; GRWR (Graft to Recipient Weight Ratio): 2,2\%; G/SLV (Graft/Standard Liver Volume): $80 \%$.

Intraoperative we found the native liver was almost dominated by a huge mass (mainly in the right liver). Mobil- 
izing the whole liver from its ligaments, we confirmed that the patient had no right hepatic vein, the middle and left hepatic vein joined into a common trunk, draining into the IVC. The superior mesenteric vein confluenced with splenic vein before draining directly into the inferior vena cava (Abernethy anomaly type 1b), just $3 \mathrm{~cm}$ below and to the left of the draining site of hepatic vein complex into the IVC. The so-called portal vein measured of $10 \mathrm{~mm}$ diameter and ran a $3 \mathrm{~cm}$ distance to the IVC (Figs. 1-3). Anatomopathology findings of the native liver showed chronic hepatitis with cirrhosis $4 / 6$ Knodel-Ishak (Fig. 4).

The immunosuppressive inducted by basiliximab, then maintained with tacrolimus and mycophenolate.

The post operative course was uneventful; the allograft function's well with normalization of total bilirubin within
3 days, and AST/ALT down under 2N (upper normal value) within 9 days. CT Scan control 3 weeks postopratively showed all hepatic vessels patent with good flow (Fig. 5). Patient was discharged 28 days postoperatively. He was doing well at the follow-up 12 months later. His mentality was much improved according to his parents.

\section{DISCUSSION}

CAPV were diagnosed in children in $80 \%$ with age ranges from the prenatal period to a 64 years. ${ }^{5}$ The extrahepatic shunts were not significant difference between male and female proportion, however male gender were found in majority of intrahepatic shunts. ${ }^{4,7}$

Sakamoto et al. ${ }^{6}$ found in published literature 34 liver transplants related to CEPS worldwide to the year 2012.
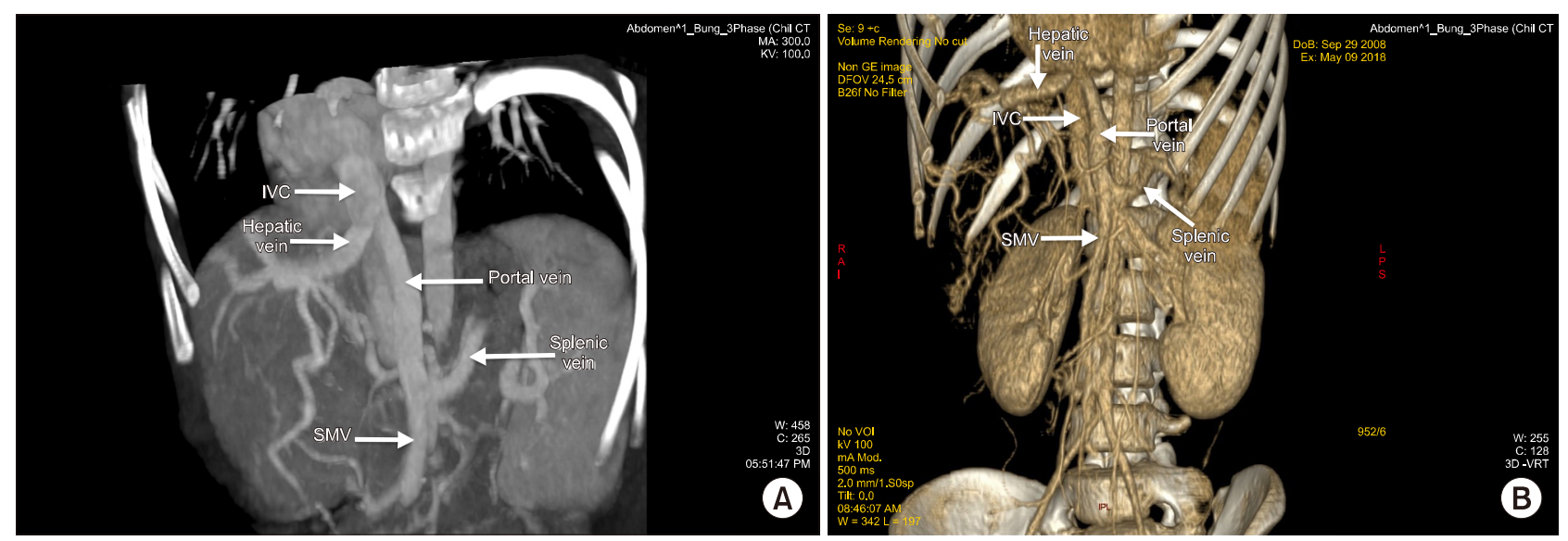

Fig. 1. (A) Abdomen 3-phase CT Scan; (B) visceral vascular 3D reconstruction showed portal vein drained into inferior vena cava in end-to-side manner.

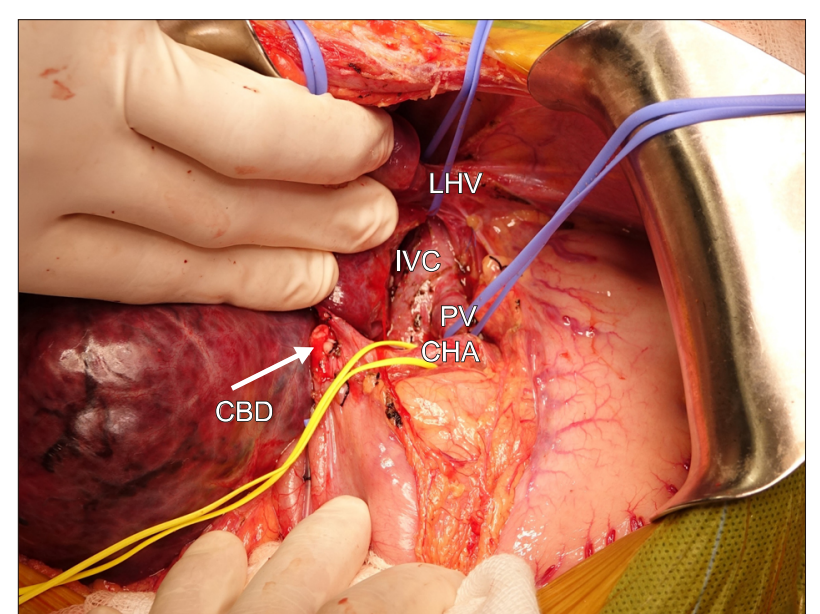

Fig. 2. Intraoperative view: portal vein drained into inferior vena cava in end-to-side manner.

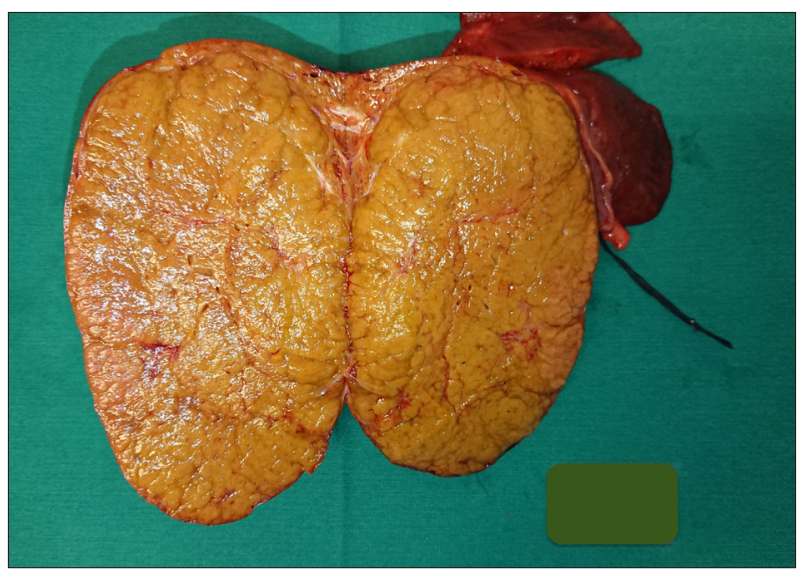

Fig. 3. Native liver macroscopic mass explanted view. 


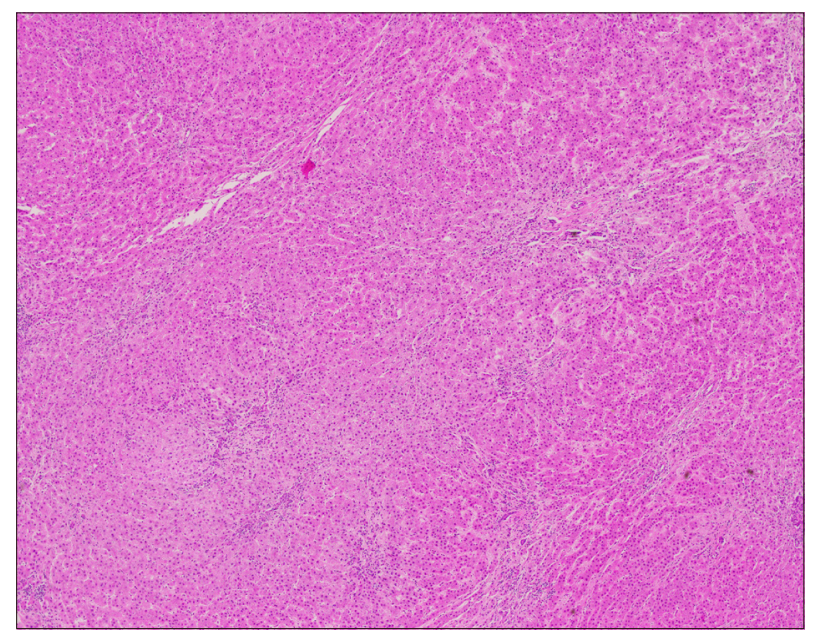

Fig. 4. Pathology findings charactered with chronic hepatitis, cirrhosis. Portal triads with arterioles and interlobular bile ducts, absence of portal central venules (H\&E stain, $\times 400)$.

Most cases were diagnosed in childhood, except for 2 cases in adulthood (amongst 13 cases (38.2\%) before one year old). Liver transplantation underwent at the age from 4 months to 45 years (mean 6.8 years). Male: female ratio was 15:19. The initial clues that lead to CEPS diagnosis were related to hepato-pulmonary syndrome or pulmonary hypertension in 9 cases $(26.5 \%)$, hypergalactosemia at neonatal screening in $9(26.5 \%)$. Six other cases $(17.6 \%)$ related to biliary atresia, while hepatic encephalopathy (HE) including mild and transient symptoms, were met in 3 cases $(8.8 \%)$. Altogether 23 cases $(67.7 \%)$ were multi-anomalies (cardiac defects, polysplenia, biliary atresia, situs inversus $\cdots)^{6}{ }^{6}$

Together with our case, worldwide literature also noted such concurrent anomalies in heart and skeletal system., ${ }^{9,10}$

This malformation presented with a wide range of clinical signs and symptoms related to the type of shunt and other concurrent anomalies. Hepatic encephalopathy syndrome (HES) in various degrees, mental retardation due to hyperammonemia, hyperbilirubinemia, hypergalactosemia; jaundice, fatigue, right upper-quadrant pain. ${ }^{6,11}$ Kobayashi et al. ${ }^{9}$ found only $13.2 \%$ CEPS related with HES. The decreased ammonia concentration and urease activity of the patient's faeces by intestinal flora were demonstrated. This acts as a homeostatic regulation, accounts for the fact that hepatic encephalopathy clinically uncommon or transient and with a mild degree. ${ }^{11-13}$ This comply with our patient who suffered mild mental defect and blood ammonia just slightly elevated.

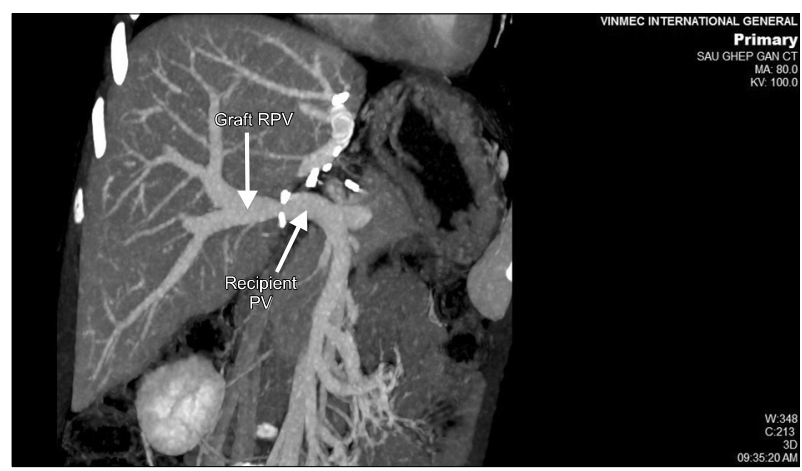

Fig. 5. Abdomen 3-phase CT Scan 3 weeks after right-lobe graft liver transplantation (Patent recipient PV as well as graft right $\mathrm{PV})$.

The liver abnormal presentation often seen with focal nodular hyperplasia, nodular regenerative hyperplasia or hemangiomas. Kobayashi et al. ${ }^{9}$ reviewed 136 cases of CEPS showing that $43 \%$ (55 cases) presented with liver tumors, and most of them benign (83.6\%). ${ }^{6,14}$ Respiratory symptoms usually seen as cyanosis, orthodeoxia platypnea associated with hepatopulmonary syndrome (HPS). ${ }^{5,15}$

Amongst 34 liver transplantations worldwidely reviewed by Sakamoto et al. ${ }^{6}$ related to CAPV, the indications due to HPS in 13 cases $(38.2 \%)$, respiration complications in $11(32.4 \%)$, liver mass in 5 (14.7\%) (2 cases with hepatoblastome and 1 hepatocellular carcinoma), 4 biliary atresia and one persistent rectal bleeding. The grafts came from 15 brain death donors and 19 living donors.

Our indication for the child based on the huge mass in the right liver and start to show deterioration of liver function (elevation of liver enzymes) related to Abernethy malformation type $1 \mathrm{~b}$.

Liver tumor in the CAPV is very common and mostly benign. Although hepatocellular carcinoma were reported by some authors, liver biopsy was not recommended routinely, though it helps clearify the malignancy as well as reveal the existence of small portal venules in the portal triads. $^{14}$

The current treatment guideline is not standardized due to scattered data and various studies used different terminology, some equating the Abernethy malformation of any type of CAPV. However most of authors consider liver transplantation for uncontrol hyperammonemia, or HES or liver masses. ${ }^{5}$ Treatment options also depends on the type of porto-caval shunt. Liver transplantation is the most effective and practical for type $1 \mathrm{CAPV}$, while in type 2 , 
cure can be ensured by surgical closure or interventional radiology (coil embolization or catheter-device closure). ${ }^{6,14}$ With potential risk of developing pulmonary hypertension and severe HPS (which preclude LT later) Brasoveanu as cited from Soejima et al. ${ }^{5}$ proposed prophylactic LT in children with CAPV.

Due to the lack of portal blood flow (accounts for $80 \%$ in normal circumstancy), it is particular finding that hepatic artery dilated in CAPV for compensation. ${ }^{1,5,16}$ In our case, the graft came from an adult (right hepatic artery diameter of $2 \mathrm{~mm}$ ) to a child (common hepatic artery diameter of $2.3 \mathrm{~mm}$ ), we successfully reconstructed the hepatic artery by manual without microscope.

Portal vein reconstruction could be extremely difficult due to the short portal conduit in some cases that require venous interposition graft. ${ }^{5}$ However in our patient the mesenterico-splenic confluence runs quite a long way enough to make a simple anastomosis (length of $3 \mathrm{~cm}$ ).

CAPV was originaly not associated with portal hypertension, therefore there was no collateral circulation. This leads to intraoperative risk of important intestinal congestion during portal clamping and anastomotic performance. $^{5,14,17}$ Sibuleski et al. ${ }^{18}$ experienced one case of intraoperative non-trauma splenic rupture due to abruptly portal hypertension while clamping the portal vein in liver transplantation. In the present case, we hold the portal phase clamping to the very last moment, just when finished the hepatic vein anastomosis and ready for a portal vein reconstruction. This helped minimize portal clamping time (to 28 minutes) without consequence on vesceral perfusion. There are other reports of portal clamping in liver transplant due to CAPV duration in 15-17 minutes without significant consequence on intestinal edema. However in theory, it should be borne in mind that mesenterical pressure might be elevated due to resistence of the new liver graft that could affect the intestinal perfusion postoperatively. ${ }^{3,5}$

Liver transplantation was a justified indication for congenital absence of the portal vein type 1, presented with huge liver parenchyme hyperplasia and liver failure. Livingdonor liver transplantation was effective and practical in the scarcity of donor organ.

\section{ACKNOWLEDGEMENTS}

The authors express thanks to Chu Chong Woo, Kwan Woo Kim, and Young Mok Park for their contribution.

\section{CONFLICT OF INTEREST}

No potential conflict of interest relevant to this article was reported.

\section{ORCID}

Hoang Duc Nam: https://orcid.org/0000-0003-1312-2413

\section{AUTHOR CONTRIBUTIONS}

Conceptualization: HDN. Writing - original draft: HDN. Writing - review \& editing: HDN.

\section{REFERENCES}

1. Abernethy J, Bank J. IX. Account of two instances of uncommon formation, in the viscera of the human body. Philos Trans R Soc 1793;83:59-66.

2. Howard ER, Davenport M. Congenital extrahepatic portocaval shunts--the Abernethy malformation. J Pediatr Surg 1997;32: 494-497.

3. Morgan G, Superina R. Congenital absence of the portal vein: two cases and a proposed classification system for portasystemic vascular anomalies. J Pediatr Surg 1994;29:1239-1241.

4. Sokollik C, Bandsma RH, Gana JC, van den Heuvel M, Ling SC. Congenital portosystemic shunt: characterization of a multisystem disease. J Pediatr Gastroenterol Nutr 2013;56:675681 .

5. Brasoveanu V, Ionescu MI, Grigorie R, Mihaila M, Bacalbasa $\mathrm{N}$, Dumitru R, et al. Living donor liver transplantation for unresectable liver adenomatosis associated with congenital absence of portal vein: a case report and literature review. Am J Case Rep 2015;16:637-644.

6. Sakamoto S, Shigeta T, Fukuda A, Tanaka H, Nakazawa A, Nosaka S, et al. The role of liver transplantation for congenital extrahepatic portosystemic shunt. Transplantation 2012;93:12821287.

7. Stringer MD. The clinical anatomy of congenital portosystemic venous shunts. Clin Anat 2008;21:147-157.

8. Tanaka Y, Takayanagi M, Shiratori Y, Imai Y, Obi S, Tateishi $\mathrm{R}$, et al. Congenital absence of portal vein with multiple hyperplastic nodular lesions in the liver. J Gastroenterol 2003;38:288294.

9. Kobayashi N, Niwa T, Kirikoshi H, Fujita K, Yoneda M, Saito $\mathrm{S}$, et al. Clinical classification of congenital extrahepatic portosystemic shunts. Hepatol Res 2010;40:585-593.

10. Takagaki K, Kodaira M, Kuriyama S, Isogai Y, Nogaki A, Ichikawa N, et al. Congenital absence of the portal vein complicating hepatic tumors. Intern Med 2004;43:194-198. 
11. Niwa T, Aida N, Tachibana K, Shinkai M, Ohhama Y, Fujita $\mathrm{K}$, et al. Congenital absence of the portal vein: clinical and radiologic findings. J Comput Assist Tomogr 2002;26:681-686.

12. Kamiya S, Taniguchi I, Yamamoto T, Sawamura S, Kai M, Ohnishi N, et al. Analysis of intestinal flora of a patient with congenital absence of the portal vein. FEMS Immunol Med Microbiol 1993;7:73-80

13. Nakasaki H, Tanaka Y, Ohta M, Kanemoto T, Mitomi T, Iwata $\mathrm{Y}$, et al. Congenital absence of the portal vein. Ann Surg 1989; 210:190-193.

14. Alonso-Gamarra E, Parrón M, Pérez A, Prieto C, Hierro L, López-Santamaría M. Clinical and radiologic manifestations of congenital extrahepatic portosystemic shunts: a comprehensive review. Radiographics 2011;31:707-722.
15. Kim MJ, Ko JS, Seo JK, Yang HR, Chang JY, Kim GB, et al. Clinical features of congenital portosystemic shunt in children. Eur J Pediatr 2012;171:395-400.

16. Pupulim LF, Vullierme MP, Paradis V, Valla D, Terraz S, Vilgrain V. Congenital portosystemic shunts associated with liver tumours. Clin Radiol 2013;68:e362-e369.

17. Shinkai M, Ohhama Y, Nishi T, Yamamoto H, Fujita S, Take $\mathrm{H}$, et al. Congenital absence of the portal vein and role of liver transplantation in children. J Pediatr Surg 2001;36:1026-1031.

18. Sibulesky L, Taner CB, Willingham DL, Satyanarayana R, Peiris P, Krishna M, et al. Orthotopic liver transplantation for hepatic adenoma in a patient with portal vein agenesis. Open Transplant J 2009;3:22-25. 